DOI: $10.3901 / J M E .2015 .14 .015$

\title{
基于温度传感标签的主轴热误差无线 监测方法及试验*
}

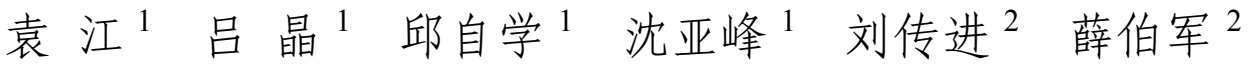

(1. 南通大学机械工程学院 南通 226019;

2. 南通国盛机电集团有限公司 南通 226002)

\begin{abstract}
摘要: 针对数控机床主轴热误差有线监测方法存在的布线困难和温度测点布置优化问题, 提出一种基于热敏区域黄金分割布 点和利用温度传感标签实现机床温度分布监测及信号无线传输的新方法。设计由数字式温度传感器 DS18B20、微处理器 MSP430、无线射频通信模块 NRF905 集成的温度传感标签, 可通过无线射频通信将温度信号及对应标签地址信息无线传输 给上位机, 通信协议简单, 能耗要求低; 根据机床主轴有限元分析模型采用黄金分割法确定了热敏感布点区域, 以解决传统 经验布点存在数据冗余、可信度不强、测试条件不确定最优等问题; 利用温度传感标签和激光位移传感器构建监测系统并采 用正交试验法进行试验测试, 数据极差分析和回归分析结果表明该系统稳定可靠、实时处理能力强, 构建的回归模型也有较 好的预测能力。
\end{abstract}

关键词: 机床主轴; 热误差; 温度传感标签; 黄金分割; 正交试验

中图分类号: TG502; TH161

\section{Wireless Monitoring Method and Experiment of Spindle Thermal Error Based on Temperature Sensor Tag}

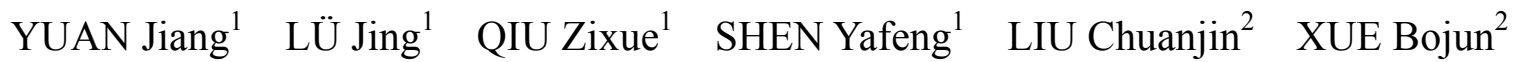 \\ (1. School of Mechanical Engineering, Nantong University, Nantong 226019;
}

2. Nantong Guosheng Electromechanical Group Company Ltd., Nantong 226002)

\begin{abstract}
To solve problems of complex cabling and temperature sensor placement optimization for CNC machine tool spindle thermal error by wired monitoring method, a new layout method of measuring points is proposed by golden section of heat sensitive area and the temperature distribution monitoring and wireless signal transmission are achieved by using temperature sensor tag. The temperature sensor tag is designed by integrating digital temperature sensor DS18B20, microprocessor MSP430, radio frequency communication module NRF905. The temperature signal and tag address information were transmitted to computer by radio frequency communication, the communication protocol is simple and energy consumption is low. According to the finite element analysis model of the machine spindle, the heat sensitive area is determined by golden section method. It can solve problems of traditional experience method such as data redundancy, unreliability, uncertain optimal test conditions and etc. The monitoring system is built by using temperature sensor tag and laser displacement sensor, and the thermal error experiment was done by the orthogonal test. The results of range analysis and regression analysis show that the system has the characteristics of reliability, strong real-time processing capability, and the regression model also has good prediction ability.
\end{abstract}

Key words: spindle; thermal error; temperature sensor tag; golden section method; orthogonal test

\section{0 前言}

在现代制造技术高速发展的今天，机床的热变

形问题已变得日趋严重 ${ }^{[1-2]}$ 。有资料研究表明, 影响 机床加工精度的误差主要是高速主轴区和导轨区发 热导致的热变形误差，占总制造误差的 $40 \%$ $70 \%{ }^{[3-5]}$ 。目前, 主轴热误差常采用试验建模法, 即 先利用温度传感器或红外测温仪测出温度数据, 同 时采用激光干涉仪或高精度激光位移传感器同步测 
出热变形数据, 然后采用最小二乘法 ${ }^{[6]}$ 、神经网 络 ${ }^{[7]}$ 、灰色理论 ${ }^{[8]}$ 、最小二乘支持向量机 ${ }^{[9]}$ 等进行热 误差分析与建模。由于温度传感器测点的选择一般 都基于工程经验, 通过安装大量传感器测试各分布 测点的温度, 再采用统计相关分析优选出少量的温 度传感器用于热误差建模分析, 这种方法存在数据 冗余、可信度不强、测试条件不确定最优等问题; 而且各个温度传感器一般是通过有线方式实现信 号传输, 众多的信号缆线给现场安装调试带来极大 不便。

融合传感器与无线射频识别 (Radio frequency identification, RFID)电子标签的传感标签技术, 不仅 可发挥无线射频技术在自动识别上的独特技术优势 实现标签地址信息的识别, 而且电子标签内嵌了传 感器具备了感知能力, 并可利用 RFID 广播通信方 式协议简单的特点, 实现分布在线监测与信号的无 线传输与处理 ${ }^{[10-13]}$ 。本文提出一种基于 RFID 传感 标签技术利用温度传感标签和读写器实现温度信 号无线采集传输的新方法, 在对主轴热敏特性分析 的基础上利用黄金分割法对热敏区域进行迭代缩 小, 以确定布置温度传感标签的最佳热敏区域, 最 后采用正交试验设计安排试验, 并利用极差法和回 归分析方法分析出最佳测试工况和热误差回归预 测模型。

\section{1 监测系统原理}

如图 1 所示, 数控机床主轴热误差监测系统 主要由温度传感标签、读写器、激光位移传感器 及控制器、USB 摄像头、监测中心组成。

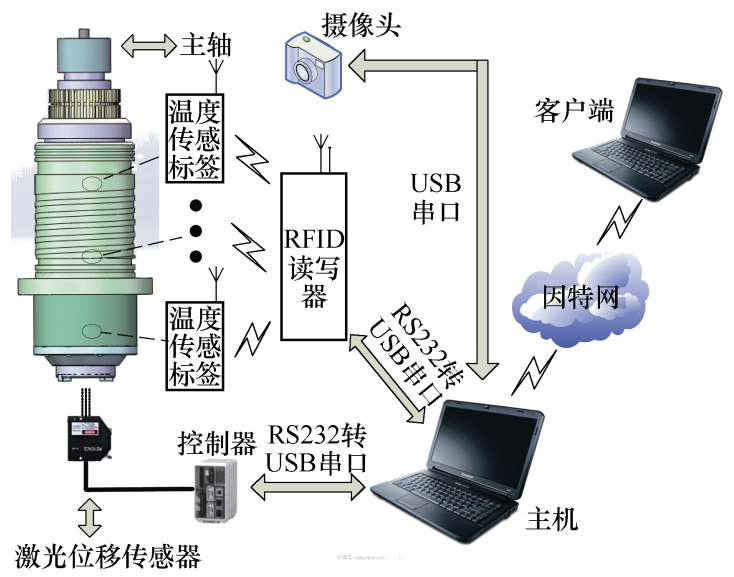

图 1 机床主轴热误差监测系统

测量时, 温度传感标签粘贴在主轴测点表面 上, 激光位移传感器置于主轴端面轴的中心延长线
上。温度传感器获取主轴分布测点的温度数据, 其 数字输出信号被传感标签内的微处理器采集, 与标 签地址信号打包后由传感标签无线传输给读写器, 读写器再将信号通过 RS232-USB 转接口传输给上 位机; 而同时激光位移传感器采集的热位移信息通 过专用的控制器传输给上位机; 上位机利用开发的 专用软件对获取的温度测点数据和热变形进行统计 相关分析。同时，监测系统的视频设备对现场工况 进行实时采集, 并会自动生成远程链接网址, 以供 管理者或客户端通过 Internet 进行远程实时监控。

\section{2 监测系统设计}

\subsection{RFID 传感标签设计}

为实现对温度数据的采集及信号的无线传输 与处理, 设计了如图 2 所示的 RFID 温度传感标签, 主要由温度传感单元、微处理器、无线收发单元、 液晶显示单元、存储单元、供电单元等组成。

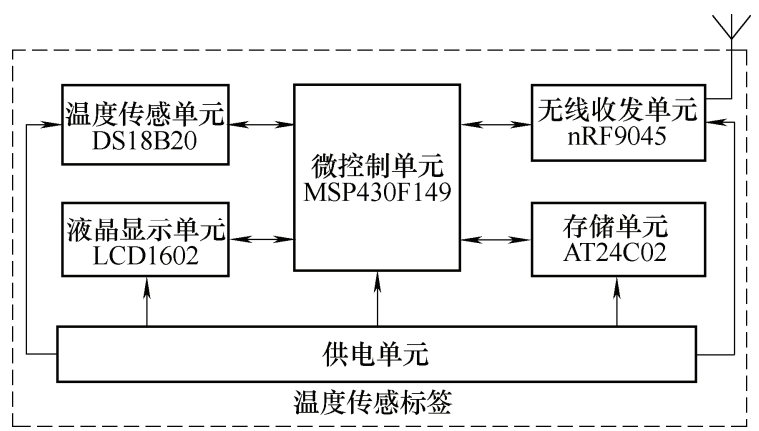

图 2 温度传感标签结构框图

温度传感单元选用美国 Dallas 公司生产的数字 化温度传感器 DS18B20, 它将温度传感器、模数转 换器、接口电路集成在一个芯片里, 具有低功耗、高 性能、抗干扰强的特点, 测温范围为 $-55 \sim+125{ }^{\circ} \mathrm{C}$, 可满足机床温度测试要求; 微控制单元选用美国德 州仪器 (TI) 公司推出的超低功耗处理器 MSP430F149, 具有低功耗、处理能力强、高性能模 拟技术及丰富的片上外围模块等特点，在满足机床 热误差测量的同时，减少了硬件电路设计的复杂度; 无线收发模块选择挪威(Nordic)公司的 NRF905, 其 工作电压为 $1.9 \sim 3.6 \mathrm{~V}$, 待机模式下耗电流仅为 2.5 $\mu \mathrm{A}$ ，可以在 $433 / 868 / 915 \mathrm{MHz}$ 开放 ISM 频段免许可 证使用; 存储单元采用美国 CATALYST 公司生产的 存储芯片 $\mathrm{AT} 24 \mathrm{C} 02$, 其是一个容量为 $2 \mathrm{kB}$ 的串行 CMOS E $22 R O M$; 液晶显示单元采用 LCD1602, 具 有体积小、显示内容丰富、超薄轻巧等优点。 


\section{2 读写器设计}

如图 3 所示, 设计的读写器包括微处理器模块、 存储模块、主控模块、供电模块、电平转换模块和 射频模块。其中，为了实现单片机与计算机之间的 串口通信, 设计时选择美国德州仪器(TI)生产的 MAX3232 芯片, 以将单片机能识别的 TTL 电平转 化为计算机能够识别的 RS-232 电平。

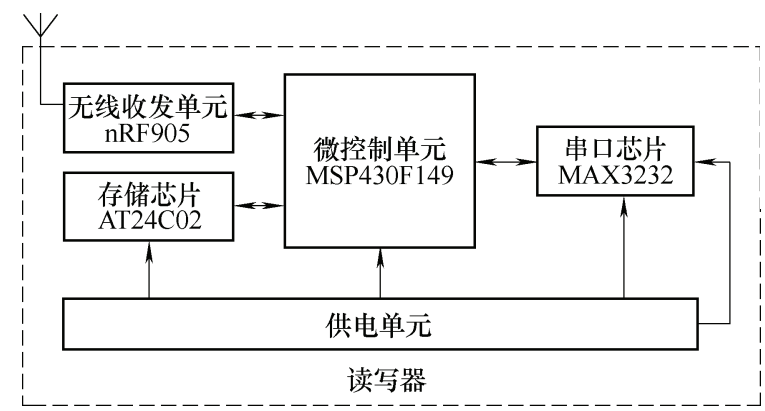

图 3 读写器结构框图

由于目前上位机中的 RS232 接口较少, 为此将 读写器输出接口利用 RS232-USB 转接头转化为 USB 通信模式, 上位机在 LabVIEW 开发平台上通 过调用 VISA 函数获取读写器串口数据。

\section{3 激光位移传感器及控制器}

机床主轴轴向的热位移测量是通过日本基恩 士(Keyence)公司的激光位移传感器 LK-G30 和专用 控制器 LK-G3001V 实现的, 具有检测光点小 (30 $\mu \mathrm{m})$ 、再现精度高 $(0.05 \mu \mathrm{m})$ 的特点, 但其一般是通 过自带的软件进行通讯、数据保存等, 这给热变形 与温度同步综合监测带来了困难, 为此, 本文通过 转接头将其控制器的网端通讯 I/O 转换成 USB 接口 模式, 并在 LabVIEW 开发平台上设计了专用的热 位移采集处理程序。

\subsection{USB 视频远程监控}

数控机床主轴热误差测试时, 操作者可根据实 际需求开启视频监测, 即采用 USB 摄像头对现场测 试工况图像进行采集。该模块利用 LabVIEW 中 IMAQ USB 函数调用摄像头实现与上位机通讯, 图 像采用 RGB 形式显示在前面板中, 同时, 通过图 像保存函数可实现实时拍照保存功能, 大大减少了 视频采集系统的成本投入。

在获取视频的基础上, 服务器端利用 LabVIEW 中的 Web Publishing Tools 将热误差监测应用程序 的前面板嵌入到 Web 页面中, 服务器端的应用程序 载入内存, 客户端便可以通过浏览器对远程的测试 状态进行监控, 客户端控制的权限也可以通过远程
面板的 Request/Release Control VI 获得或释放。这 种 Web 远程发布具有跨平台、易配置、操作简单等 特点, 为数控机床热误差远程监控提供了便利。

\section{5 上位机软件设计}

采用美国 NI 公司图形化开发平台 LabVIEW 进 行了上位机系统软件的设计。如图 4 所示, 先登陆 用户、机床型号等信息, 然后初始化配置读写器、 激光位移传感器、USB 视频设备等参数, 测量时通 过上位机同步发送采集命令, 测量分布测点的温度 数据和机床主轴轴向热变形数据, 最后上位机对获 取的温度测点数据和热变形数据进行统计相关分 析，实现机床热误差建模分析及预测，同时，客户 端可以通过服务器生成的链接进行远程监测控制。

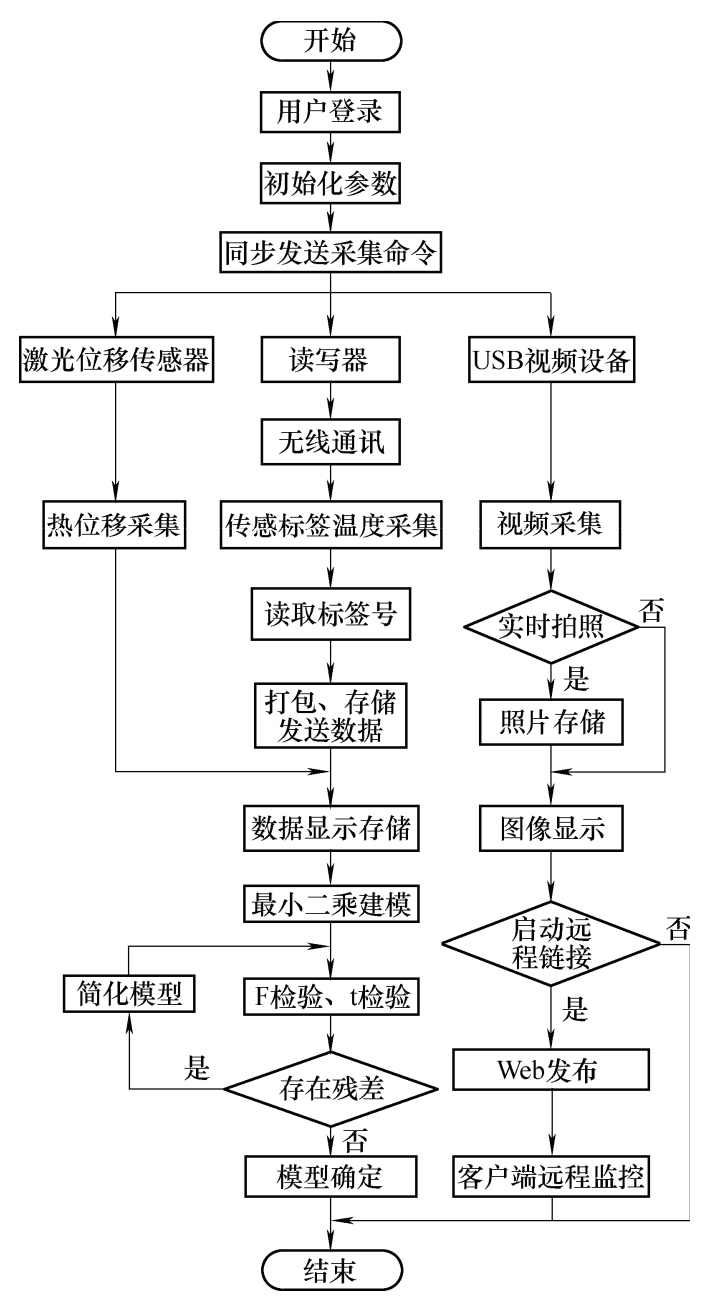

图 4 系统软件流程图

\section{6 系统测量精度试验}

对监测系统所采用的温度传感标签和激光位 移传感器分别进行了精度测量试验。

选取台湾普森(POSA)主轴, 转速保持 3000 $\mathrm{r} / \mathrm{min}$, 运转 $90 \mathrm{~min}$, 每隔 $2 \mathrm{~min}$ 采样一次, 采用设 计的 RFID 传感标签(图 5a)和高精度红外测温仪(图 
5b)进行了温度对比试验, 结果如图 6、7 所示。

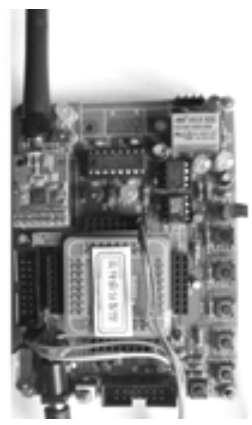

(a) 温度传感标签

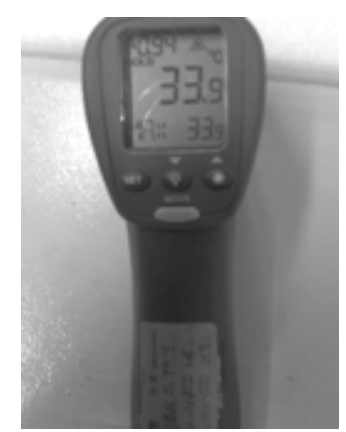

(b) 红外测温仪
图 5 温度测量设备

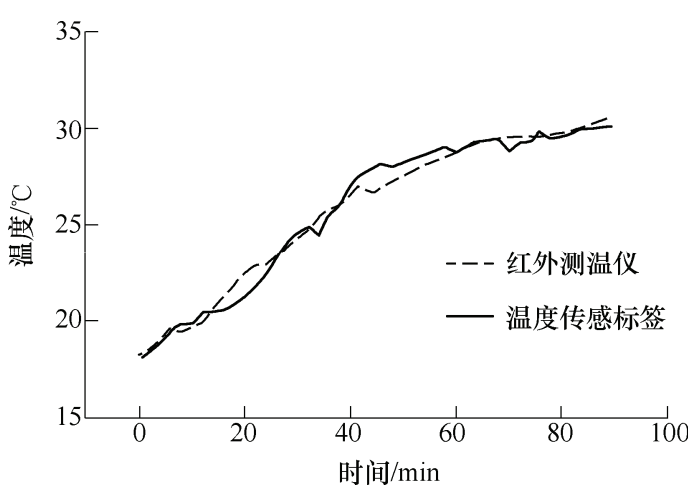

图 6 温度传感标签与红外测温仪的测试结果

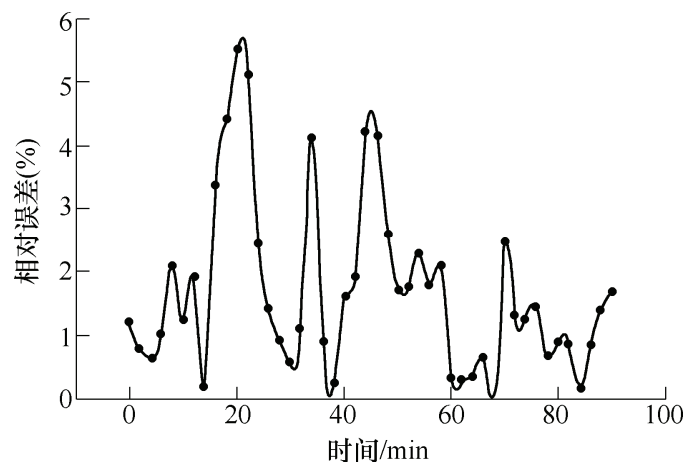

图 7 温度传感标签与红外测温仪的相对误差

从图 6 可以看出设计的温度传感标签与红外测温 仪测试趋势基本相近, 图 7 为两种测量方法的相对误 差, 从图 7 可以看出两者测试结果最大相对误差为 $5.53 \%$, 平均相对误差为 $1.69 \%$, 这说明由温度传感 标签和读写器组成的无线传输系统可实现对温度信 号的正确获取与无线传输, 并具有较高的测量精度。

采用日本基恩士的激光位移传感器 LK-G30 和 英国雷尼绍的激光干涉仪 XL-80 同时对主轴轴向进 行位移测试, 由于激光位移传感器测距范围为 25 $35 \mathrm{~mm}$, 故控制主轴底端端面距激光位移传感器初 始距离为 $25 \mathrm{~mm}$, 并逐次递增 $1 \mathrm{~mm}$ 进行数据记录, 结果分别如图 8、9 所示。

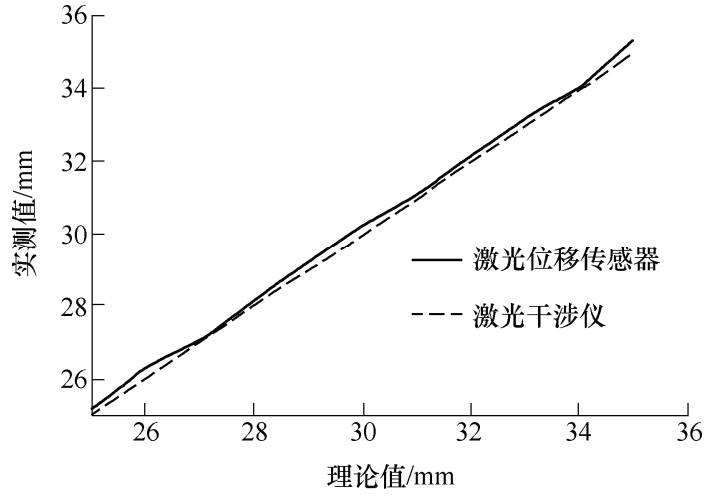

图 8 激光位移传感器与激光干涉仪的测试结果

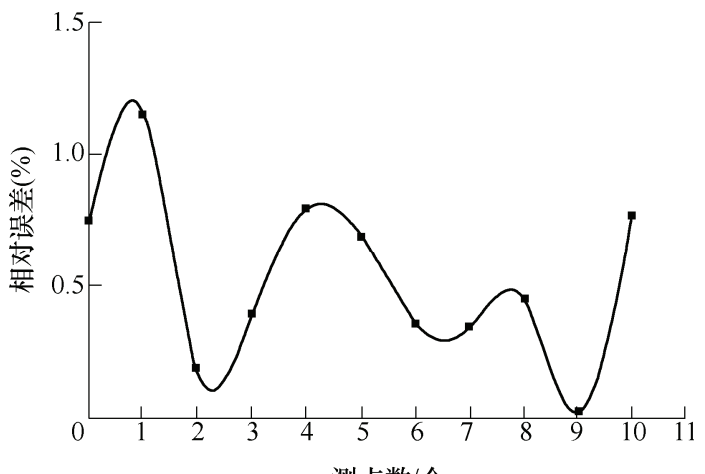

测点数 $/$ 个

图 9 激光位移传感器与激光干涉仪的相对误差

从上述结果可以看出, 激光位移传感器与激光 干涉仪最大相对误差为 $1.16 \%$, 平均相对误差为 $0.54 \%$, 这说明激光位移传感器测量精度较高, 满 足热位移测试需求。

\section{3 温度测点布置优化}

由于机床热变形误差在很大程度上取决于加 工条件、加工周期、冷却液的使用以及周围环境等 多种因素，因此温度测点的布置、优化选择，对热 误差建模与补偿影响很大 ${ }^{[14-17]}$ 。为此, 本文从机床 的传热特性出发，提出一种基于热敏区域黄金分割 的测点布置优选方法。

\section{1 热敏区域黄金分割法}

如图 10 所示，首先对主轴进行热敏特性分 析, 包括结构模型简化、热边界条件确定、温度 场及热变形仿真分析, 在此基础上用 ANYSYS WORKBENCH 中的 Probe 指针根据主轴热态分析 云图值标注热变化敏感位置并结合主轴三维尺寸, 初步量化出主轴热敏区域 $[a, b]$, 然后在此区间上将 第一个分割点 $x_{1}$ 安排在试验范围内的 0.618 处, 即 $x_{1}=a+(b-a) \times 0.618$, 再在 $x_{1}$ 的对称点 $x_{2}=b-(b-a) \times 0.618$ 处进行分割试验, 并以两次试验的温度与热变形之 
间相关系数作为筛选依据, 保留相关性较大的分割 点, 然后在保留区间内继续插入第三个分割点, 以 此类推逐渐缩小热敏区间, 直至迭代至适宜区间为 止, 最终确定出主轴黄金热敏区域。

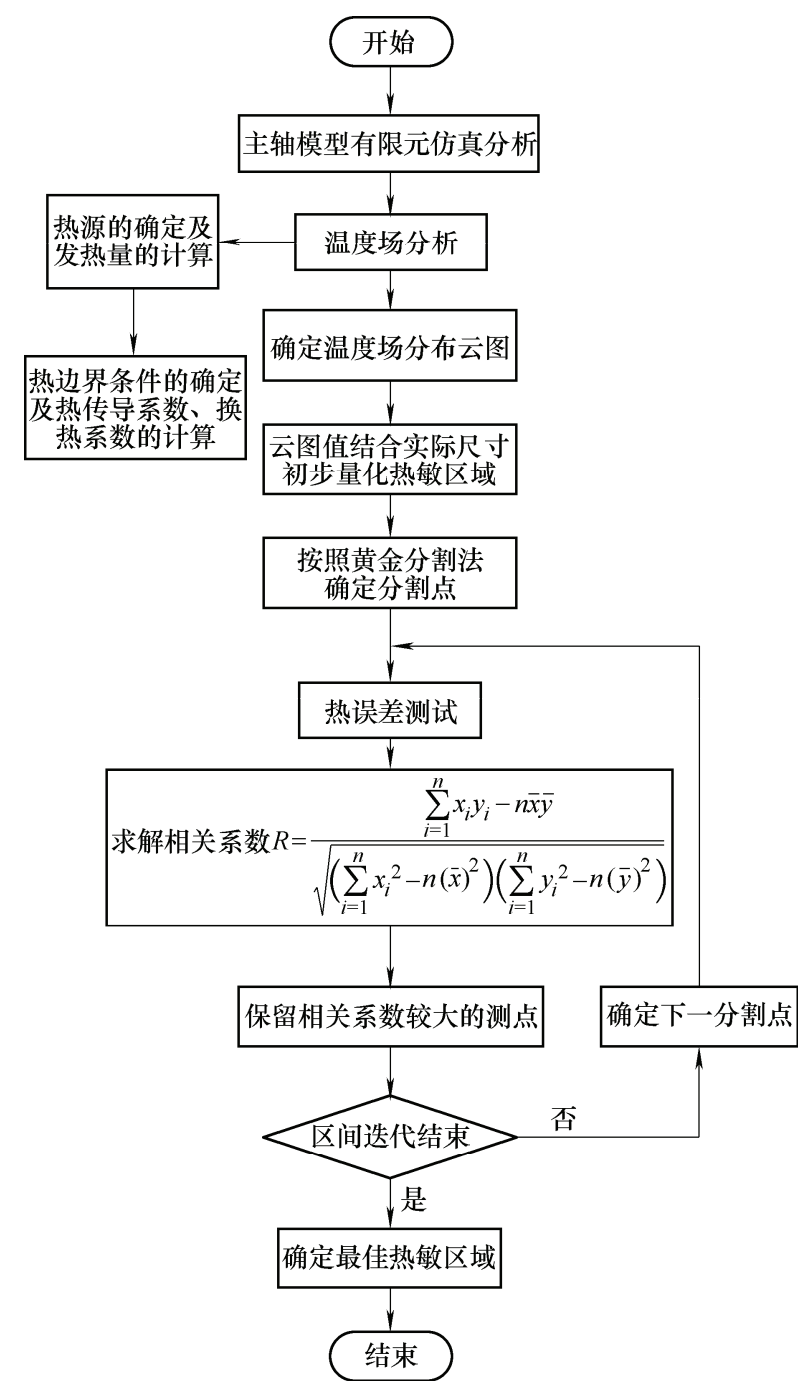

图 10 热敏区域黄金分割法流程图

\section{2 区域量化及分割}

选取台湾罗翌(ROYAL)公司的某型号主轴, 其 轴长为 $515 \mathrm{~mm}$, 直径为 $150 \mathrm{~mm}$ 。根据上述步骤, 初步确定热敏区域范围为 $[35 \mathrm{~mm}, 165 \mathrm{~mm}]$ 。在此区 域内, 沿主轴轴向采用黄金分割法缩小热敏区域, 并以分割点的相关系数作为优选依据, 考虑到主轴 实际尺寸及现场传感器的布置需求, 确定轴线区域 缩小至 $(30 \pm 1) \mathrm{mm}$ 为宜, 算法运行结果如表 1 所示。

从表 1 可以看出, 当分割第 4 个测点后, 搜索 区间的范围变为 $[53.97 \mathrm{~mm}, 84.66 \mathrm{~mm}]$, 区间跨度为 $30.69 \mathrm{~mm}$, 满足迭代结束条件, 停止搜索, 同时为 方便现场划分布点区域, 确定测点分布的最佳热敏 区域为 $[54 \mathrm{~mm}, 84 \mathrm{~mm}]$ 。
表 1 最佳热敏测点求解结果

\begin{tabular}{cccc}
\hline 分割序号 & 分割点位置 $/ \mathrm{mm}$ & 相关系数 & 搜索区间 $/ \mathrm{mm}$ \\
\hline 1 & $x 1=115.34$ & 0.7264 & $(35,165)$ \\
2 & $x 2=84.66$ & 0.7686 & $(35,115.34)$ \\
3 & $x 3=65.69$ & 0.9012 & $(35,84.66)$ \\
4 & $x 4=53.97$ & 0.8606 & $(53.97,84.66)$ \\
\hline
\end{tabular}

\section{3 测点布置}

将主轴热敏区域沿某一轴切线进行切割展开成 矩形, 并在矩形区域内划分网格按照均匀布点原则 进行布点, 考虑到主轴轴向是主要散热方向, 布点 间隔应较小，而对于圆周向，散热较均匀，布点间 隔可尽量大些。如图 11 所示, 在热敏区域内布置了 四个测点, 横向间隔取 $90 \mathrm{~mm}$, 纵向间隔取 $10 \mathrm{~mm}$ 。

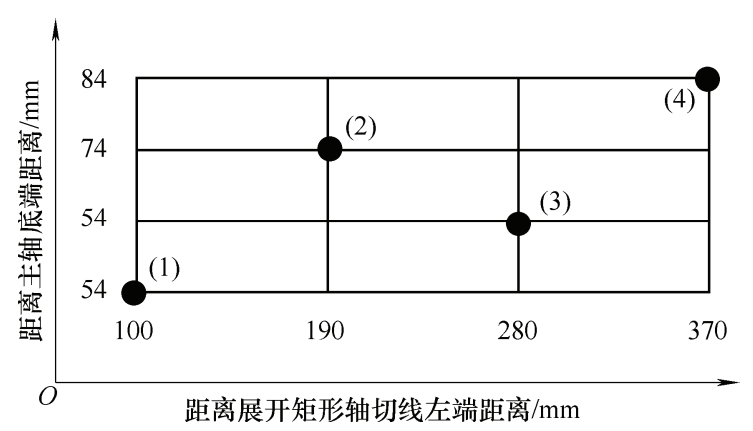

图 11 测点布置图

\section{4 正交试验及数据分析}

为使测试工况达到最优, 同时减少试验次数, 采用正交试验设计方法安排测点温度及热变形误差 测试, 并分别采用极差分析法和回归分析法以确定 热误差测试优方案、各因素主次顺序以及回归预测 模型。

\section{1 正交试验设计}

选取转速、时间和有无冷却为试验设计因素, 各因素各取 2 个水平，正交设计表采用 $L_{4}\left(2^{3}\right)$ ，构 件建的因素水平表如表 2 所示。

表 2 因素水平表

\begin{tabular}{cccc}
\hline 水平 & 时间 $/ \mathrm{min}$ & 转速 $/(\mathrm{r} / \mathrm{min})$ & 有无冷却 \\
\hline 1 & 90 & 4000 & 有 \\
2 & 120 & 6000 & 无 \\
\hline
\end{tabular}

\section{2 试验数据极差分析}

根据正交设计测点布置, 对每个测点依次进行 试验, 测试完毕, 将结果导入自主开发的相关系数 程序中进行求解, 并利用极差法进行分析, 求解结 果如表 3 所示。 
表 3 正交试验结果

\begin{tabular}{cccccccc}
\hline $\begin{array}{c}\text { 试验 } \\
\text { 序号 }\end{array}$ & $\begin{array}{c}\text { 时间/ } \\
\min \end{array}$ & $\begin{array}{c}\text { 转速/ } \\
(\mathrm{r} / \mathrm{min})\end{array}$ & $\begin{array}{c}\text { 有无 } \\
\text { 油冷 }\end{array}$ & 测点 1 & 测点 2 & 测点 3 & 测点 4 \\
\hline 1 & 90 & 4000 & 有 & 0.7619 & 0.8801 & 0.8769 & 0.7705 \\
2 & 90 & 6000 & 无 & 0.8567 & 0.9826 & 0.9413 & 0.8812 \\
3 & 120 & 4000 & 无 & 0.8347 & 0.9455 & 0.9279 & 0.8294 \\
4 & 120 & 6000 & 有 & 0.7945 & 0.9294 & 0.8954 & 0.8227 \\
\hline
\end{tabular}

由表 3 可以看出, 同一测点在不同工况下, 测 点温度与热变形之间的相关系数是变化的, 4 个测 点的相关系数的大小变化趋势一致; 而且, 在表中 任一工况下, 测点 2 和测点 3 的相关系数要高于测 点 1 和测点 4 , 因此, 可将测点 2 和测点 3 作为主 轴热敏感测点。

对热敏测点 2 的测试数据, 采用极差法分析影 响热误差相关性最显著的工况及各因素的主次关 系，三个因素极差数据分析结果如表 4 所示。

表 4 极差分析结果

\begin{tabular}{ccccc}
\hline 因素号 & 试验因素 & 相关系数和 $K_{1}$ & 相关系数和 $K_{2}$ & 极差 $r$ \\
\hline 1 & 时间 $/ \mathrm{min}$ & 1.8627 & 1.8749 & 0.0122 \\
2 & 转速 $/(\mathrm{r} / \mathrm{min})$ & 1.8256 & 1.9120 & 0.0864 \\
3 & 有无冷却 & 1.8095 & 1.9281 & 0.1186 \\
\hline
\end{tabular}

表 4 中 $K_{1}$ 表征的是因素 1(时间)、因素 2(转速) 和因素 3(有无冷却)在 1 水平下各自的相关系数之 和; $K_{2}$ 为因素 1(时间)、因素 2(转速)和因素 3(有无 冷却)在 2 水平下各自的相关系数之和; $r$ 称之为极 差, 其计算公式为 $r=\max \left(K_{1}, K_{2}\right)-\min \left(K_{1}, K_{2}\right)$ 。

由表 4 可以看出 $r_{3}>r_{2}>r_{1}$, 这说明冷却液对主 轴热误差测试影响最为显著; 此外, 对于各因素的 $K_{2}$ 均大于 $K_{1}$, 这表明在主轴热误差测试时, 主轴的 油冷会减小热变形及热误差之间的相关度, 因此, 为更好地研究温度与热变形之间的变化关系, 增强 后续构建模型的可信度, 测试时, 最优方案为运转 时间取 $120 \mathrm{~min}$, 转速 $6000 \mathrm{r} / \mathrm{min}$, 且无需冷却。

\section{3 回归建模及检验}

对四个布置测点的测试结果进行热误差建模, 并利用 $\mathrm{F}$ 检验法、 $\mathrm{t}$ 检验法分别衡量模型的可靠性 以及各测点的显著性。设温度变化值与热变形量之 间满足近似函数关系式为

$$
y=a+b_{1} x_{1}+b_{2} x_{2}+b_{3} x_{3}+b_{4} x_{4}
$$

式中, $x_{1} 、 x_{2} 、 x_{3} 、 x_{4}$ 为测点温度变化值; $y$ 为热变 形值; $a 、 b_{1} 、 b_{2} 、 b_{3} 、 b_{4}$ 为回归系数。

将测试结果 $x_{1 i} 、 x_{2 i} 、 x_{3 i} 、 x_{4 i}$ 代入式(1)便可 得到对应的函数计算值 $y_{i}$, 根据最小二乘法原理, 建立正规方程组, 求解即得回归方程系数 $a 、 b_{1}$ 、 $b_{2} 、 b_{3} 、 b_{4}$ 。为简化求解过程, 利用 LabVIEW 图形 化函数设计了快速、简捷的回归分析程序, 并构建
方差分析与偏回归分析表, 结果如图 12 所示。

\begin{tabular}{|c|c|c|c|c|c|}
\hline & 主轴测点多元 & $\overrightarrow{\mathrm{\tau}}$ 线性回归分 & 析一南通大学 & 机械工路学院 & 8 \\
\hline \multirow{3}{*}{$\mathbf{y}=\frac{\mathbf{a}}{-0.00191}$} & \multirow[b]{2}{*}{ b1 } & \multicolumn{2}{|c|}{ 回归方程 } & \multirow[b]{2}{*}{ b3 } & \multirow[b]{2}{*}{ b4 } \\
\hline & & \multicolumn{2}{|c|}{ b2 } & & \\
\hline & +0.000297 & $\mathrm{X}_{1+} \quad 0.00$ & $2795 \mathrm{X}_{2+}$ & $-0.0007 \cdot \mathrm{X}_{3+}$ & $0.00034 \mathrm{X}_{4}$ \\
\hline & & \multicolumn{2}{|c|}{ 方差分析表 } & & \\
\hline 差异源 & SS & HS & $\mathrm{F}$ & $\mathrm{F}_{0.01}$ & 显著性 \\
\hline 回归 & $0.0029 !$ & 0.000737 & 19. 9593 & 3. 83 & **** \\
\hline 残差 & 0.00147 & $3.6957 \mathrm{E}-$ & & & \\
\hline \multirow[t]{3}{*}{ 总和 } & 0.00442 & & & & \\
\hline & & \multicolumn{2}{|c|}{ 偏回归系数 $\mathrm{t}$ 检验 } & & \\
\hline & $\mathrm{t}$ & & $\mathrm{t}$ & to. 05 & to. 01 \\
\hline $\mathrm{x} 1$ & 0.84491 & $\mathrm{x} 3$ & -2.9415 & & \\
\hline $\mathrm{x} 2$ & 9. 67466 & $\mathrm{X} 4$ & 1.30475 & & \\
\hline
\end{tabular}

图 12 回归模型分析结果

由图 12 可以看出, 在给定显著水平 0.01 下, 回归方程 $F>F_{0.01}$, 这说明该回归方程非常显著。同 时, 在对偏回归系数进行 $\mathrm{t}$ 检验时, $|t|$ 越大, 所对 应的偏回归系数越显著, 相应的因素越重要, 由 $\mathrm{t}$ 检验结果可知, 测点的主次顺序为 $x_{2}>x_{3}>x_{4}>x_{1}$, 且 $\left|t_{2}\right|>\left|t_{3}\right|>\left|t_{0.01}\right|>\left|t_{0.05}\right|>\left|t_{4}\right|>\left|t_{1}\right|$, 因此, 测点 1 、 测点 4 对试验结果影响不显著, 可将这两项并到残 差中, 即回归方程变为二元线性方程

$$
y=0.0028 x_{2}-0.0007 x_{3}-0.0019
$$

式中, $x_{2} 、 x_{3}$ 为测点 $2 、 3$ 位置处的温度变化值; $y$ 为对应的热变形值。

对上述方程再进行回归分析, 结果如图 13 所示。

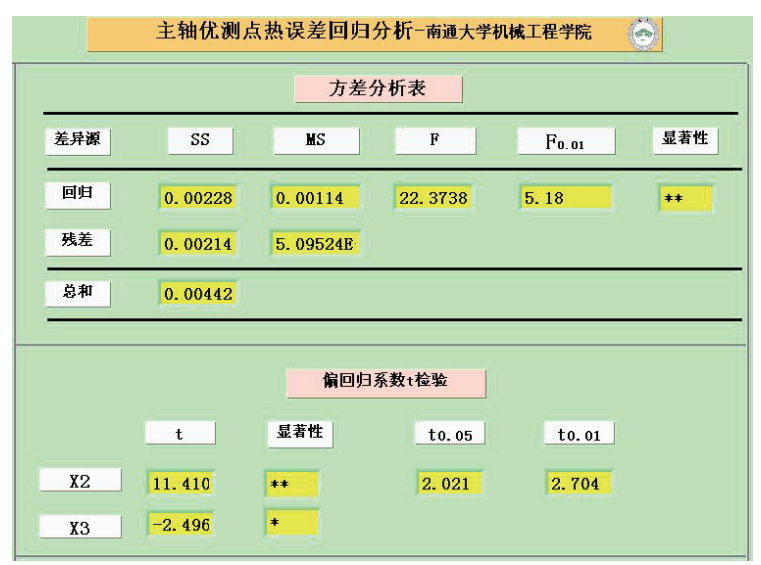

图 13 简化后的模型回归分析结果

由图 13 可知, 在给定显著水平 0.01 下, 回归 方程 $F>F_{0.01}$, 这说明简化后的回归方程高度显著; 由 $\mathrm{t}$ 检验结果可知, $\left|t_{2}\right|>\left|t_{0.01}\right|>\left|t_{3}\right|>\left|t_{0.05}\right|$, 测点 2 的偏回归系数高度显著, 测点 3 的偏回归系数也比 较显著, 这说明在热敏点 $2 、 3$ 处监测的温度与机床 主轴热变形之间有显著的线性关系, 用这两点的测 试结果构建的回归模型有较好的预测能力。 


\section{5 结论}

(1) 设计了一种具有感知与标识功能的温度传 感标签, 可将采集的温度数据与标签地址编码信息 通过射频通信无线传输给读写器和上位机, 传感标 签与读写器之间通信协议简单, 功耗低, 且测量精 度与红外测温仪相当。

(2) 为快速缩小布点区域, 提出一种基于热敏 区域黄金分割的布点区域搜索算法, 在主轴温度分 布云图的基础上, 结合实际尺寸初步量化热敏测点 区间，利用黄金分割优选法对其迭代缩小，最终确 定热敏区域为 $[54 \mathrm{~mm}, 84 \mathrm{~mm}]$ 。

(3) 采用正交试验设计方法在主轴热敏区域内 安排试验, 通过极差分析法确定出测试优方案, 对 测点处的结果建立最小二乘回归模型, 并通过 $\mathrm{t}$ 检 验法将原来的 4 个测点减少到 2 个, 简化后的回归 模型可靠、预测性较好, 为实现快速误差补偿奠定 了基础, 具有一定的推广应用价值。

\section{参 考 文 献}

[1] VYROUBAL J. Compensation of machine tool thermal deformation in spindle axis direction based on decomposition method [J]. Precision Engineering, 2012, 36 (1): 121-127.

[2] 陈松, 王永青. 数控机床热误差实时补偿研究 [J].机械 设计与制造, 2013, 9(9): 53-55.

CHEN Song, WANG Yongqing. The research of CNC thermal error real time compensation[J]. Machinery Design \& Manufacture, 2013, 9(9): 53-55.

[3] WANG Y C, KAO M C, CHANG C P. Investigation on the spindle thermal displacement and its compensation of precision cutter grinders[J]. Measurement, 2011(44): 1183-1187.

[4] 仇健, 刘春时, 刘启, 等. 龙门数控机床主轴热误差及 其改善措施[J]. 机械工程学报, 2012, 48(21): 149-157. QIU Jian, LIU Chunshi, LIU Qi, et al. Thermal errors of planer type NC machine tools and its improvement measures[J]. Journal of Mechanical Engineering, 2012, 48(21): $149-157$.

[5] CREighton E, HONEGGER A, TUlsian A, et al. Analysis of thermal errors in a high-speed micro-milling spindle[J]. International Journal of Machine Tools \& Manufacture, 2010, 50(4): 386-393.

[6] 雷春丽, 茌执元. 基于多元自回归模型的电主轴热误 差建模与预测 [J]. 机械科学与技术, 2012, 31(9):
1526-1529.

LEI Chunli, RUI Zhiyuan. Thermal error modeling and forecasting based on multivariate autoregressive model for motorized spindle[J]. Mechanical Science and Technology, 2012, 31(9): 1526-1529.

[7] 苏宇锋, 袁文信, 刘德平, 等. 基于 $\mathrm{BP}$ 神经网络的电 主轴热误差补偿模型 $[\mathrm{J}]$. 组合机床与自动化加工技术, 2013(1): 36-38.

SU Yufeng, YUAN Wenxin, LIU Deping, et al. A thermal errors compensation model for high-speed motorized spindle based on BP neural network[J]. Modular Machine Tool \& Automatic Manufacturing Technique, 2013(1): 36-38.

[8] 李永祥, 童恒超, 杨建国. 灰色系统理论在机床热误 差测点优化中的应用 [J]. 机械设计与研究，2006, 22(3): 78-81.

LI Yongxiang, TONG Hengchao, YANG Jianguo. Application of grey system theory in optimizing the measuring points of thermal erron on machine tools[J]. Machine Design and Research , 2006, 22(3): 78-81.

[9] 姜辉, 杨建国, 姚晓栋, 等. 数控机床主轴热漂移误差 基于贝叶斯推断的最小二乘支持向量机建模[J]. 机械 工程学报, 2013, 49(15): 115-121.

JIANG Hui, YANG Jianguo, YAO Xiaodong, et al. Modeling of $\mathrm{CNC}$ machine tool spindle thermal distortion with LS-SVM based on Bayesian inference[J]. Journal of Mechanical Engineering, 2013, 49(15) : 115-121.

[10] 江昌旭, 王磊, 李桂香, 等. 基于 RFID 的无线多目标 温湿度监控系统[J]. 仪表技术与传感器, 2013(5): 45-47. JIANG Changxu, WANG Lei, LI Guixiang, et al. Wireless multi-objective temperature and humidity monitoring system based on RFID $[\mathrm{J}]$. Instrument Technique and Sensor, 2013(5): 45-47.

[11] LUCA C, RICCARDO C, ALESSANDRA E, et al. RFID sensor-tags feeding a context-aware rule-based healthcare monitoring system[J]. Journal of Medical Systems, 2012, 6(36): 3435-3449.

[12] 袁江, 邱自学, 邵建新, 等. 基于无线射频识别传感标 签的形状记忆合金增强复合结构低速冲击响应监测 [J]. 机械工程学报, 2012, 48(18): 89-96.

YUAN Jiang, QIU Zixue, SHAO Jianxin, et al. Low speed impact test for shape memory alloy-reinforced composite structure based on radio frequency identification sensor-tags $[\mathrm{J}]$. Journal of Mechanical Engineering, 2012, 48(18): 89-96. 
[13] STEINBERG I M, STEINBERG M D. Radio frequency tag with optoelectronic interface for distributed wireless chemical and biological sensor applications[J]. Sensor and Actuators B: Chemical, 2009, 138(1): 120-125.

[14] 高峰, 刘江, 杨新刚, 等. 基于 Fisher 最优分割法的机 床热关键点优化研究 [J]. 仪器仪表学报, 2013, 34(5): 1070-1075.

GAO Feng, LIU Jiang, YANG Xingang, et al. Study on optimization of thermal key points for machine tools based on Fisher optimal segmentation method[J]. Chinese Journal of Scientific Instrument, 2013, 34(5): 1070-1075.

[15] 张琨, 张毅, 侯广锋, 等. 基于热模态分析的热误差温 度测点优化选择 $[J]$. 机床与液压, 2012, 40(7): 1-3.

ZHANG Kun, ZHANG Yi, HOU Guangfeng, et al. Selection of sensor placement for thermal error compensation based on thermal mode analysis[J]. Machine Tool \& Hydraulics, 2012, 40(7): 1-3.

[16] LI Y X, YANG J G, GELVIS T, et al. Optimization of measuring points for machine tool thermal error based on grey theory $[\mathrm{J}]$. International Journal of Advanced Manufacturing Technology, 2008, 35(7): 745-750.

[17] 林伟青, 傅建中, 许亚洲, 等. 基于 LS-SVM 与遗传算 法的数控机床热误差辨识温度传感器优化策略 $[\mathrm{J}]$. 光 学精密工程, 2008(9): 1682-1687.

LIN Weiqing, FU Jianzhong, XU Yazhou, et al. Optimal sensor placement for thermal error identification of $\mathrm{NC}$ machine tool based on LS-SVM and genetic algorithm[J]. Optics and Precision Engineering, 2008(9): 1682-1687.

作者简介: 袁江, 男, 1968 年出生, 副教授。主要研究方向为机电系统 装备及测控技术。

E-mail: yuanjiang68@126.com

吕晶, 男, 1989 年出生。主要研究方向为虚拟仪器技术。

E-mail: 539921220@qq.com

邱自学, 男, 1963 年出生, 博士, 教授, 博士研究生导师。主要研究方 向为智能结构健康监测技术。

E-mail: qiu.zx@ntu.edu.cn 